Relations industrielles

Industrial Relations

\title{
Analyse critique de la Loi des relations ouvrières et du Règlement no 1 de la C.R.O. (II)
}

\section{Alfred Charpentier}

Volume 16, numéro 2, avril 1961

URI : https://id.erudit.org/iderudit/1021801ar

DOI : https://doi.org/10.7202/1021801ar

Aller au sommaire du numéro

Éditeur(s)

Département des relations industrielles de l’Université Laval

ISSN

0034-379X (imprimé)

1703-8138 (numérique)

Découvrir la revue

Citer cet article

Charpentier, A. (1961). Analyse critique de la Loi des relations ouvrières et du Règlement no 1 de la C.R.O. (II). Relations industrielles / Industrial Relations, 16(2), 206-237. https://doi.org/10.7202/1021801ar
Résumé de l'article

La première tranche de cette étude a été publiée dans la livraison précédente de cette revue. La deuxième qui suit se divise en six parties : a) conventions collectives, b) pratiques interdites, c) devoirs des assotiations, d) la Commission des relations ouvrières, e) de la grève illégale et ses responsabilités, f) observations diverses.
Tous droits réservés @ Département des relations industrielles de l’Université Laval, 1961
Ce document est protégé par la loi sur le droit d'auteur. L'utilisation des services d'Érudit (y compris la reproduction) est assujettie à sa politique d'utilisation que vous pouvez consulter en ligne.

https://apropos.erudit.org/fr/usagers/politique-dutilisation/ 


\title{
Analyse critique de la Loi des relations ouvrières et du Règlement no 1 de la C.R.O. (II)
}

\author{
Alfred Charpentier
}

La première tranche de cette étude a été publiée dans la livraison précédente de cette revue. La deuxième qui suit se divise en six parties: a) conventions collectives, b) pratiques interdites, c) devoirs des assotiations, d) la Commission des relations ouvrières, e) de la grève illégale et ses responsabilités, f) observations diverses.

\section{Conventions collectives}

\section{Avis de Négociation}

Selon l'article 11 l'«association reconnue» qui avise l'autre partie de son désir de la rencontrer pour la conclusion d'une convention collective, ce n'est nulle autre ordinairement que l'association des salariés. Plusieurs employeurs ignorent tel avis même si, comme le veut l'article 11, l'association y demande la rencontre après un délai de huit jours en précisant le jour, l'heure et le lieu.

Un employeur résolument réfractaire à l'article 11 de la Loi manifeste son refus de négocier. Devant telle situation l'attitude de la C.R.O. jusqu'au début de 1960, était la suivante: la Commission devait s'assurer si l'association avait servi à l'employeur l'avis prescrit à l'article 11; sinon, elle lui ordonnait de s'y conformer avant de considérer subséquemment, s'il y avait lieu, sa demande d'intervention conciliatrice. Mais si l'association avait observé l'article 11 la Commission envoyait à l'employeur une mise en demeure d'avoir à négocier. S'il refusait encore de rencontrer l'association, la Commission livrait alors les deux parties entre les mains du conciliateur. Si l'employeur refusait aussi lintervention de ce dernier la Commission autorisait l'association à le traduire en justice ou elle recommandait au Procureur général de le poursuivre pour lui faire imposer les pénalités prévues par la Loi.

A cette procédure lente et temporisatrice a peut-être succédé une procédure prompte et énergique depuis les modifications draconiennes apportées, il y a un an, aux articles 42 et 49 de la L.R.O. touchant l'augmentation des pénalités et le droit de poursuite à la C.R.O. pour toute violation à la Loi. 


\section{DEUX TYPES DE CONVENTIONS PARTICULIEkRES}

Si les négociations s'entament normalement entre une association ouvrière et un employeur le type de convention collective prévu ordinairement par la Loi des relations ouvrières est la convention particulière à une entreprise, soit qu'elle s'applique à un seul établissement ou à plusieurs établissements similaires du même employeur.

Par une interprétation libérale de la Loi, la C.R.O. a permis l'introduction, dans le champ d'opération de la Loi, d'un deuxième type de convention particulière, c'est la convention de base (master agreement) conclue coinjointement par plusieurs entreprises similaires et ensuite individualisée à chaque entreprise par une déclaration personnelle de chacun des employeurs contractants. C'est le type de convention particulière négociée indirectement par le truchement et l'action conjointe d'une association patronale et d'une centrale professionnelle ouvrière. Depuis environ 7 ans il est maints exemples de ce type de convention de base individualisée.

La possibilité de procéder comme ci-dessus en matière de négociation collective entre deux associations patronale et ouvrière est sujette à déroger dans certains cas, de la règle imposée par la C.R.O. d'individualiser une convention de base. La première dérogation de ce genre la Commission l'a constatée incidemment en avril 1958 dans le domaine de l'enseignement scolaire. C'est le cas d'une convention collective de base qui a été conclue entre un syndicat d'instituteurs diocésains et une fédération diocésaine de commissions scolaires, qui n'a pas été ensuite particularisée à chaque commission scolaire à titre d'employeur distinct. Non seulement le cas présent n'est pas conforme aux exigences de la Loi des relations ouvrières mais il ne répond pas non plus à la même exigence de la Loi concernant les corporations municipales et scolaires et leurs employés.

\section{Cadre de la convention}

\section{Durée de la convention}

Par le cadre de la convention collective nous voulons dire les quatre clauses qui conditionnent la vie d'une convention, savoir: durée, dénonciation, prolongation et revision.

Le premier paragraphe de l'article 15 lorsqu'il fut amendé, il y a quelques années, pour permettre d'étendre la durée d'une convention collective à deux ou trois ans est inconciliable avec la Loi des corporations municipales et scolaires qui impose une durée de deux ans à toute convention collective entre tels organismes et leurs employés. Ce n'est qu'une incompatibilité théorique, mais qui existe vu que cette dernière loi est subordonnée à la L.R.O., en vertu de laquelle se font 
reconnaitre les associations de salariés des corporations municipales et scolaires. Reconnaissance qui leur confère le pouvoir de forcer les négociations collectives et de jouir de maintes autres prérogatives émanant de la Loi des relations ouvrières, sauf en ce qui a trait à la durée de la convention, à son renouvellement automatique, à la procédure de l'arbitrage et à l'interdiction du droit de grève. Ajoutons une autre disposition propre à la Loi des corporations municipales et scolaires, c'est le droit d'insérer dans une convention collective une clause de rajustement automatique du salaire pendant la durée de la convention.

\section{Dénonciation de la convention}

Le deuxième paragraphe de l'article 15 indique la procédure à suivre pour éviter le renouvellement automatique d'une convention collective d'un an. La C.R.O. décida, appuyée par les tribunaux, que l'avis écrit donné par l'une des deux parties à l'autre dans le délai stipulé à l'effet de ne plus être liée par ladite convention collective à son expiration, pouvait être servi suivant l'un ou l'autre des trois énoncés à la fin dudit paragraphe 15, à savoir: «y mettre fin ou la modifier, ou en négocier une nouvelle ${ }^{1}$. A moins que dans la convention un autre mode spécifique à ce sujet y soit stipulé, l'emploi de l'un ou l'autre des trois modes précités est considéré par la C.R.O. comme avis de dénonciation de la convention.

\section{Convention intérimaire}

L'article 15 dans son dernier paragraphe, prévoit la possibilité pour les parties contractantes de prolonger de quelques mois la convention en cours s'ils ne sont pas prêts à la renouveler le jour de son expiration. Cela peut se faire au moyen d'un simple engagement mutuel à cette fin ou par voie d'une convention intérimaire de moins d'un an. Mais ceci, a décidé la Commission, ne peut fermer le délai légal prévu à l'article 16 permettant entre le $60 \mathrm{e}$ et le $30 \mathrm{e}$ jour précédant la fin d'une convention, la présentation d'une requête en certification par une association intervenante.

\section{Clause de revision}

Souvent une convention collective contient une clause de réouverture pour rajustement de salaire pendant sa durée (après six mois ou un an selon le cas). Cette pratique assez courante non prévue dans la L.R.O. nécessite parfois, pour effectuer une entente, le recours à l'inter-

(1) Voir dans « Recueil annoté des lois ouvrières » de Gérard Vaillancourt, p. 155, une décision majoritaire de la C.R.O. (D-27) dans Wabasso Cotton Co. Ltd. et syndicat national des employés de Wabasso Cotton, de Shawinigan Falls. 
vention conciliatrice et à l'arbitrage, ou, dans le cas contraire, le statuquo continue ou les parties sont conduites à la grève ou au lockout. Procédures de conciliation et d'arbitrage auxquelles le ministère du Travail doit se prêter de bonne grâce en dehors de la Loi en pareille occurrence. Peu importe le temps écoulé avant que toutes ces procédures ne soient terminées, même si elles dépassent le terme de la convention originale, la Commission ne ferme pas le délai donnant droit, s'il y a lieu, à l'intervention d'un tiers à la date d'expiration de ladite convention.

\section{DÉPỐT DE LA CONVENTION}

\section{a) selon larticle 19}

L'article 19 de la Loi exige le dépôt à la C.R.O. de deux copies certifiées de la convention qui a été conclue. Il incombe à l'association de salariés d'effectuer ce dépôt, non à l'employeur comme cela est arrivé parfois. En pareil cas la Commission devait demander à l'association d'approuver le dépôt. Lorsque la convention est conclue par deux associations (patronale et ouvrière) les deux associations, au sens de l'article 19, doivent chacune déposer copie de leur convention. Habituellement seule l'association ouvrière fait ce dépôt. La C.R.O. s'en satisfait.

Lorsque le dépôt d'une convention est effectué par une centrale professionnelle ou centrale syndicale mandatée à cette effet par l'association locale certifiée, la centrale ouvrière doit s'assurer que les parties signataires sont bien les personnes concernées dans le certificat de reconnaissance syndicale qui a été émis ou que ce sont bien les personnes dûment mandatées.

Il arrive qu'une centrale ouvrière et une association patronale concluent une convention de base( «master agreement») et désirent conjointement la rendre particulière à l'établissement de chaque employeur membre de l'association patronale, alors une déclaration écrite en ce sens doit être faite par chacun d'eux; bien entendu, ces dépôts de conventions particulières ne sont obligatoires que pour les établissements vis-à-vis desquels un certificat de reconnaissance a déjà été émis par la C.R.O.

Il est cependant important de noter qu'il arrive parfois que l'on omet le dépôt d'une convention collective. Ce qui prive notamment l'association ouvrière reconnue des recours de la Loi des relations ouvrières.

Lorsque le Service de dépôt des conventions collectives de la C.R.O. reçoit la copie d'une convention collective, il s'assure, avant d'en effectuer le dépôt, que ladite convention est conforme à l'article 15 de la L.R.O. ou aux dispositions de la Loi concernant les corporations 
municipales et scolaires, dans le cas des associations touchant les employés de ces organismes.

Si la convention n'est pas conforme à l'une ou l'autre des dispositions des lois susdites, elle est retournée aux parties intéressées accompagnées d'un avis signalant l'irrégularité, ou les irrégularités à corriger à savoir: l'identité des signataires, la durée de la convention et la clause de renouvellement automatique ou non.

Le dépôt ne compte qu'une fois la convention collective corrigée et retournée à la C.R.O.

Rien dans la L.R.O. n'oblige les parties contractantes d'aviser la C.R.O. si la convention a été abrogée ou dénoncée, si elle a été renouvelée automatiquement sans amendements ou avec amendements, si elle est remplacée par une nouvelle convention, enfin si elle est en cours de négociation. La Commission a besoin d'obtenir ces renseignements eu égard aux délais légaux à être observés dans l'application des articles 15 et 16 , advenant l'intervention d'une tierce partie.

La Commission a aussi besoin de ces renseignements pour juger de la justification de la demande d'un employeur de reviser l'effectif ouvrier représenté par un certificat de reconnaissance syndicale antérieurement émis.

Afin d'obtenir les renseignements plus haut signalés le secrétariat de la C.R.O. doit communiquer périodiquement avec les associations certifiées au moyen d'une formule officielle.

\section{b) selon larticle 19 a)}

La Loi des Syndicats professionnels adoptée en 1924, sera vingt ans plus tard inadaptée aux besoins du temps et ne conservait plus que le mérite particulier de faciliter l'incorporation civile d'une association. Par contre la Loi des relations ouvrières adoptée alors offrait des avantages supérieurs pour faciliter la négociation de conventions collectives ainsi que la procédure de conciliation et d'arbitrage. En plus la Loi des relations ouvrières primait sur la Loi des Syndicats professionnels par sa règlementation du droit de grève et du lockout. Or tous les syndicats incorporés de l'époque se sont fait reconnaftre en vertu de la Loi des relations ouvrières et cela a continué depuis.

Mais en 1944 quand fut votée la Loi des relations ouvrières, le législateur ne put prévoir si aucune disposition de cette loi ne viendrait en conflit avec un article quelconque de la L.S.P. C'est ce qui devait arriver concernant le dépôt de la convention collective. Ce dépôt allaitil être fait chez le ministre du Travail comme le requiert l'article $23 \mathrm{de}$ la L.S.P. ou allait-il être fait en vertu de l'article 19 de la L.R.O.? 
Suivant l'article 23 de la L.S.P. une copie de la convention doit être envoyée au ministre du Travail si le signataire est un syndicat incorporé; l'envoi peut être effectué par le syndicat intéressé ou par l'employeur signataire. Par contre en vertu de l'article 19 de la Loi des relations ouvrières (avant son amendement par l'article 19a) une copie de la même convention devait être également déposée à la C.R.O. par le mème syndicat incorporé, étant donné par ailleurs sa certification par la C.R.O.

Pour obvier à l'obligation de ce double dépôt de la convention collective par une association à la fois « incorporée 》 et * certifiée > il fallut donc bientôt amender la Loi des relations ouvrières, en y insérant l'article 19a qui dispense l'association incorporée de transmettre à la C.R.O. copie de la convention déposée chez le ministre du Travail, c'est le ministre qui, alors, fait cette transmission à la C.R.O. Cependant nombre de syndicats incorporés continuent à déposer leur convention collective a la C.R.O. seulement. La C.R.O. doit alors en transmettre copie au ministre du Travail.

Sous ce chef du dépôt de la convention collective il s'est révélé une autre incompatibilité entre les deux lois susdites. Aucune des dispositions de la Loi des Syndicats professionnels ne fixe la durée que peut avoir la convention collective de travail. Selon la L.R.O. sa durée (article 15) peut être d'un an, deux ans ou trois ans, et il n'est prévu de renouvellement automatiquement que pour la convention dont le terme est d'un an. Or il arrive fréquemment qu'une convention est déposée au ministère du Travail dont le terme contrevient à l'article 15 de la L.R.O. quant à la durée de sa mise en vigueur. Telle convention étant par ailleurs conforme auxx dispositions de la Loi des Syndicats professionnels, les officiers du ministère du Travail émettent automatiquement un certificat de dépôt à l'envoyeur, (association ou employeur) de la convention. Puis suit la formalité (art. 19a) de la transmission à la C.R.O. de la copie de telle convention collective.

Mais se fondant sur l'expérience des difficultés créées à la C.R.O. par la transmission de documents du genre en violation de l'article 15 de la Loi des relations ouvrières, le ministère du Travail, depuis quelques années, use d'une certaine procédure pour tenter d'obvier à ces difficultés. Lorsqu'il émet un certificat de dépôt lors de la réception d'une convention collective, il l'accompagne d'un avis à l'envoyeur (de la convention) que l'acceptation dudit dépôt est sous réserve que la convention est trouvée conforme aux exigences de la Loi des relations ouvrières par la C.R.O. Voici, après les préliminaires appropriés dans chaque cas, le texte, qui importe, du deuxième paragraphe dudit avis:

< Le dépôt de cette convention est accepté sans préjudice aux pouvoirs de la Commission de relations ouvrières de la refuser si ladite convention n'est pas conforme aux dispositions de la Loi des relations ouvrières. Deux copies certifiées sont transmises à la Commission. ? 
Cette acceptation sous réserve par le ministre du Travail permet à la C.R.O. d'obtenir des parties intéressées les corrections voulues à la durée d'une convention collective en rapport avec l'article 15 de la L.R.O. avant d'en accueillir à son tour le dépôt. Mais que vaut cette condition posée, en marge de la Loi, par le ministre du Travail si les parties négligent de faire les corrections nécessaires? La convention n'en restet-elle pas moins en vigueur selon l'article 23 de la Loi des Syndicats professionnels? En l'occurrence la Commission n'émet pas pour son compte de certificat de dépôt, (mais retient quand même la convention); cependant elle ne la considère pas en vigueur au sens de la L.R.O. Alors l'absence de convention ou tout comme, peut permettre, à la faveur d'une scission au sein de l'association ouvrière concernée, que surgisse, à un moment donné, une association intervenante qui tente de supplanter la première. Et la C.R.O. pourrait-elle refuser de recevoir sa requête en reconnaissance syndicale, eu égard à l'article 16 de la L.R.O., et cela même bien avant le terme de la susdite convention? D'autre part les parties déjà liées par une convention collective en vertu de la Loi des Syndicats professionnels ne pourraient-elles pas prétendre à la priorité de l'article 23 de ladite loi quant à la date de son expiration? ${ }^{2}$

Il arrive assez souvent qu'une convention soit déposée par la partie patronale. La Loi des Syndicats professionnels le permet, non pas la Loi des relations ouvrières. Alors dans ce cas comme dans l'autre la C.R.O. doit demander à la partie syndicale d'approuver ce dépôt par une lettre de sa part.

\section{Conciliation et arbitrage}

\section{Procédures obligatoires}

Dans l'impossibilité de conclure une convention collective, après avoir négocié en conformité aux exigences de la Loi (art. 11 et 12), l'intervention conciliatrice peut être demandée par l'une des parties à la C.R.O. Telle demande d'intervention doit indiquer les sujets du projet de convention sur lesquels l'on ne s'est pas entendu et ceux sur lesquels il y a eu entente. En l'absence de ces renseignements la Commission attend de les obtenir avant de réferer ladite demande au Service de conciliation.

Toute requête pour intervention conciliatrice doit être présentée sous la signature de la partie qui l'envoie et dont le nom apparaît sur le certificat de reconnaissance qui a été émis ou s'il y a lieu, la requête doit mentionner le nom du mandataire dûment nommé.

(2) Un cas typique de cette situation fut, en 1957, celui de Canadian Celanese Co. Ltd. et Association des employés de C.C. vs. Union des Ouvriers du Textile d'Amérique, local 1534. La Commission disposa de ce cas par une décision majoritaire. Voir textes de cette décision et de la dissidence dans le procès-verbal de la C.R.O., juin 1957, page 9195 et suivantes. 
En possession des renseignements dont il est parlé plus haut le conciliateur est en mesure d'accélérer la conciliation entre les parties. Après les quatorze jours de délai que lui accorde l'article 14 poir faire rapport il arrive souvent que le conciliateur ne puisse faire qu un rapport incomplet et qu'avec l'assentiment des parties il puisse ccntinuer ses offices auprès d'eux jusqu'à la signature d'une entente. Si, au contraire, après ce stade prolongé de la conciliation (ou même avant telle prolongation) le conciliateur doit attester de l'insuccès de ses efforts au ministre, ce dernier nomme alors un conseil d'arbitrage suivant les dispositions de la Loi des Différends ouvriers.

Or ce renvoi à la Loi des Différends ouvriers ne vaut que pour la procéduce à suivre pour l'institution d'un conseil d'arbitrage. Les huit "différends » mentionnés dans cette loi, sauf un (voir à l'article 4 de la Loi des Différends ouvriers), ne s'appliquent pas ici. Puis, contraire ment à cette dernière loi, la L.R.O. n'exige pas un minimum de dix employés à son emploi pour être considéré employeur. Il s'agit de lunité de travail reconnue.

La Loi des Différends ouvriers, qui date de 1909, ne comporte qu'une procédure facultative de conciliation et d'arbitrage qui, jusqu'en 1944, n'avait servi que pour le règlement de différends qui naissaient d'ententes passées de gré à gré ou de conventions collectives conclues en vertu de la Loi des Syndicats professionnels ou encore en vertu de la Loi de la convention collective qui pourvoit à l'extension juridique. Or c'est le recours seulement à la procédure d'arbitrage indiquée à la section III de la Loi des Différends ouvriers que l'article 14 de la L.R.O. a rendu obligatoire.

Advenant que les parties contractantes aient dû s'en remettre au prononcé d'une sentence arbitrale pour tenir lieu d'une convention collective, telle sentence arbitrale est-elle assimilable à une convention collective quant à la durée et au délai d'expiration au sens de la L.R.O.? Non, la C.R.O. a décidé qu'une sentence arbitrale ne peut s'assimiler à une convention collective quant au délai d'intervention prévue par les articles 16 et 26 de la L.R.O. ${ }^{3}$

\section{Arbitrage privé des griefs}

Il arrive qu'une clause dans une convention collective prévoit l'arbitrage privé pour le règlement d'un grief survenant pendant la durée de la convention. Généralement, la convention collective prévoit l'arbitrage d'un grief par une procédure à plusieurs paliers entre les parties conduisant à soumettre leur grief à un conseil d'arbitrage formé par le ministre du Travail. Si telle disposition est absente d'une con-

(3) Voir ouvrage cité, p. 175, une décision de la C.R.O. (D-43) re: La Commission de Transport de Montréal et deux associations rivales de ses employés. 
vention, la Loi des relations ouvrières impose l'arbitrage obligatoire par le truchement de la Loi des Différends ouvriers.

Un grief relevant de l'application d'une convention collective n'a pas besoin d'être soumis à la C.R.O. pour en demander l'arbitrage, l'on a qu'à s'adresser directement au ministère du Travail. Maintes associations passent encore par la C.R.O. sans nécessité.

\section{PRIVILÈge DE L'ASSOCIATION MINORITAIRE}

Concernant la présence d'une association minoritaire non reconnue dans une entreprise, mais présence permise par l'article 17, la Commission ne fut amenée à donner une interprétation positive de cet article qu'en 1952 en statuant que: « tout ce qu'il donne à une association autorisée à s'en prévaloir, c'est de pouvoir soumettre par écrit à l'employeur tout grief résultant d'une violation de la L.R.O. ou de la convention collective de travail. Ce qui oblige alors l'employeur de convoquer immédiatement le représentant de l'association qui est partie à la convention collective ainsi que le représentant qui a soumis les griefs pour procéder à l'examen de ces griefs sans autre recours additionnel \$.

L'article 17 a amené la C.R.O. à émettre occasionnellement une attestation d'association minoritaire à toute association en droit de le demander. C'était ordinairement une attestation pour une durée indéterminée. En fin d'année 1956 la Commission dut modifier cette ligne de conduite lorsqu'une association certifiée lui demanda de reviser l'effectif de l'association minoritaire pendant la durée de la convention collective en cours. Et ce fut le 24 juillet de l'année suivante que la Commission adopta la règle suıvante pour l'application à l'avenir de l'article 17:

\section{REVISION D'EFFECTIF}

«La C.R.O. pour le maintien de la paix de la stabilité dans l'industrie et dans les relations patronales ouvrières, a décidé que lorsqu'une attestation minoritaire a été émise, conformément aux dispositions de larticle 17 de la Loi, elle ne pourra être revisée sur requête d'une partie intéressée qu'après un délai d'un an à compter de la date de l'émission d'une telle attestation, et ce lorsqu'il n'y a pas de convention collective de travail en vigueur. Dans le cas où une convention collective de travail est intervenue entre une compagnie et une association reconnue, cette attestation minoritaire pourra être revisée, toujours sur requête d'une partie intéressée, que dans les délais prévus aux articles 16 et 26 de la Loi des relations ouvrières». (Décision de la C.R.O. re Price Bros. Co. vs International Brotherhood of Paper Makers, 24 juillet 1957.)

D'aucuns prétendent que la Loi ne stipulant pas de délai pour la revision, la partie certifiée peut l'exiger chaque fois que l'association minoritaire soumet un grief. 
Le plus gros grief dont puisse se plaindre une association minoritaire dans une entreprise c'est l'abstention ou le refus de l'association reconnue d'examiner les griefs de ladite association minoritaire en présence de son représentant et de l'employeur. Une plainte du genre fut soumise à la C.R.O. pour la première fois en 1957. En pareil cas la Commission ne peut que mettre l'association certifiée en demeure de se conformer aux prescriptions de l'article 17 et d'autre part donner a lassociation minoritaire, si elle le requiert, la permission de poursuivre l'association certifiée, si elle n'obtempère pas à la susdite mise en demeure.

A l'occasion d'une demande adressée à la C.R.O. d'enjoindre une association certifiée de donner copie de sa convention collective à l'association minoritaire, la Commission n'a pas jugé que la Loi l'autorisait à le faire.

Les griefs de l'association minoritaire relativement à la violation de la Loi des relations ouvrières regardent très particulièrement les articles touchant les «pratiques interdites », à la Section IV de la Loi.

Avant que ne soit conclue une convention collective entre l'association reconnue et l'employeur, la C.R.O. peut-elle accorder une attestation de syndicat minoritaire? Elle ne peut accueillir une requête à cette fin que lorsque a été «conclue» une convention comme le laisse bien entendre le contexte de l'article 17. La présentation d'un grief par le groupe minoritaire est subordonné à l'existence d'une convention.

Tout autre grief du groupe minoritaire relatif à la violation de la Loi en l'absence d'une convention collective ne peut être jugé, à mon sens, que par la C.R.O.

Convention par une association non reconnue

Voyons le cas de la convention négociée et conclue librement en vertu de l'article 18 de la Loi.

Que vient faire l'article 18 dans la présente Loi? Il n'est qu'une affirmation platonique du droit légitime de la négociation libre et volontaire en fait de convention collective avec son corollaire légitime du droit à la conciliation et à l'arbitrage volontaire. Ce qui implique aussi le consentement mutuel des parties à être liées, le cas échéant, par une décision arbitrale.

Telle reconnaissance du droit à la négociation volontaire, sans la coercition exercée par la loi, présuppose encore que l'employeur est moralement satisfait du caractère représentatif de l'association de ses salariés. 
L'employeur qui, à cet égard, n'est pas plus exigeant qu'il ne faut, se rencontre peu souvent. Peu souvent aussi, voit-on l'association ouvrière, tout comme l'employeur, accepter volontairement l'arbitrage et surtout se conformer volontairement à une décision arbitrale. Etat d'esprit plus manifeste de part et d'autre, semble-t-il, depuis l'application de la Loi des relations ouvrières.

L'article 18 n'offre pas la sécurité ni la tranquillité désirées dans les relations contractuelles. Son introduction dans la Loi des relations ouvrières en 1944 servait alors de trait d'union entre le régime des relations contractuelles volontaires et le régime des relations contractuelles obligatoires qui a suivi. Il y a quinze ans dans les premières années du présent régime coercitif de négociation, l'article 18 avait son utilité pour assurer la validité des conventions collectives conclues sous le précédent régime de négociation volontaire et dont le terme se prolongeait encore pour une certaine durée. Aujourd'hui l'article 18 est le plus désuet de la L.R.O., vu la croissance énorme, depuis la passation de cette loi, de la défiance patronale à l'endroit des syndicats ouvriers, d'une part, et le redoublement d'agressivité de ces derniers à l'endroit du patronat, d'autre part.

\section{Toutefois l'article 18 peut-il offrir encore quelque utilité?}

Il a été donné à la Commission de relations ouvrières de constater son application ces dernières années, en certaines circonstances singulières. Il s'agit premièrement d'associations qui quelques temps après avoir déclaré la grève contre une entreprise avant d'être certifiées par la C.R.O. purent, néanmoins, obtenir un règlement de grève par la conclusion d'une convention collective avec l'employeur. ${ }^{4}$ En deuxième lieu il s'agit de deux associations qui après avoir été décertifiées par suite de grèves illégales purent chacune, après un certain temps, obtenir un règlement de grève par la signature aussi d'un contrat de travail avec chaque employeur. ${ }^{5}$

Cas très exceptionnels que ceux-là; fruits d'une médiation très spéciale. Ententes qui ne sont évidemment que transitoires jusqu'à une éventuelle recertification des associations concernées. Pourquoi l'article 18 (avec son principe de la négociation collective volontaire) ne pourraitil être utilisé plus fréquemment ne fut-ce que pour des ententes transitoires ou provisoires? Deux circonstances surgissent fréquemment où cela pourrait être fait:

1-Dans le cas d'un nouvel établissement industriel où l'installation mécanique de la production n'est pas suffisamment avancée ou

(4) En 1957 chez Carrier \& Frères Ltée, de Shawinigan Falls.

(5) En 1959 chez Rosita Hosiery Mills Ltd, de Montréal et à l'Hôpital du SacréCoeur, de Hull. D'autres exemples seraient à citer. 
achevée pour permettre la fabrication complète du produit ou des produits dudit établissement;

2-Dans le cas d'un nouvel établissement industriel dont la production initiale n'est qu'à titre d'expérimentation pour une période de $\mathrm{X}$ mois avant d'être mise sur le marché de la concurrence. Période d'attente à être déterminée par la C.R.O. dans chaque cas particulier. Ententes provisoires dont il n'y aurait pas lieu de déposer copie à la C.R.O.

Mais, alors, pour négocier telle convention provisoire, l'association ouvrière et l'employeur doivent s'approcher dans un esprit de grande compréhension. Les deux parties doivent se consentir de mutuelles concessions et reléguer la discussion de tout sujet de désaccord sérieux après le terme de la convention provisoire, soit au moment où après avoir été certifié le syndicat pourra entamer avec l'employeur une nouvelle négociation de convention collective sous le couvert, cette fois, de tous les droits et obligations de la Loi des relations ouvrières.

Il importe de créer dans les milieux patronaux et ouvriers l'ambiance psychologique qui permettrait de tirer un meilleur profit de l'article 18.

Changement de PRopriÉtaire d'UNE ENTREPRise

Lorsque pendant la durée d'une convention une entreprise change de nom à la suite d'une vente, l'association ouvrière déjà reconnue doitelle se faire reconnaître à nouveau vis-à-vis le nouvel employeur? Un tel cas s'est présenté en 1954. La Commission acquiesça à la demande de l'association en cause, d'amender son certificat de reconnaissance vis-à-vis le nouvel employeur, après avoir obtenu l'assentiment de ce dernier et l'engagement écrit quil acceptait les obligations de la convention collective en vigueur.

Une association intervenante interjeta appel contre cette décision. La Cour renversa la décision de la Commission. La Commission porta la cause en Cour Supérieure, celle-ci, confirmée par la Cour d'Appel, jugea (en 1957) que la C.R.O. avait juridiction pour décider dans ce cas sans dire si elle avait ou non raison de décider comme elle le fit. La procédure imposée par la Cour en pareil cas oblige donc l'association à se faire reconnaître vis-à-vis le nouveau propriétaire de l'entreprise même si ce dernier a assumé les obligations de la convention déjà en vigueur.

(6) Voir Gérard Vaillancourt, op. cité pour trois décisions de la C.R.O., nos 178, 179 et 193, re: Brown Corporation, La Tuque et Canadian International Paper Co. 
Lorsqu'une entreprise change sa raison sociale seulement, sans passer à un nouvel acquéreur, la Commission n'a qu'à constater le fait et à modifier le certificat de reconnaissance.

Dans les instances où il y a objection au changement par la partie opposée, la C.R.O. fait enquếte, étudie les objections et décide au mérite.

\section{Protiques interdites}

DOMINation D'Une association de SALARú́s

Selon l'article 20 aucun employeur ne doit «chercher d'aucune manière à dominer ou entraver la formation ou les activités d'une association de salariés ». Rarement un employeur agira-t-il de la sorte personnellement. Son gérant ou un contremaître agira ainsi à sa place.

D'une façon ou d'une autre les employés apprennent que l'employeur préfère une «union intérieure » à une union extérieure; une augmentation soudaine des salaires est accordée; l'octroi d'un bonus annuel est annoncé; diverses autres améliorations sont effectuées ou annoncées; certains officiers de l'association en formation se voient offrir des avantages particuliers; permission est accordée aux employés de se réunir dans l'atelier en temps utile; parfois même l'avocat employé pour aider l'association a été suggéré par l'employeur. Voilà, à titre d'exemple, diverses façons par lesquelles un employeur peut dominer la formation d'une association «intérieure» dans son établissement et ensuite entraver ses activités.

Mais lorsqu'un employeur entrave les activités légitimes d'une association née de la seule volonté de ses employés, son acte est plus odieux; son hostilité antisyndicale est plus manifeste. Et cet entrave aux légitimes activités ${ }^{7}$ d'une association libre est un acte d'une fréquence inquiétante. L'entrave que fait un employeur contre un syndicat libre, dit de l'« extérieur», se manifeste dès sa formation par des « manières» de faire bien différentes que celles qu'emploie celui qui veut aider aे former une association de \& boutique * chez lui.

Ici l'employeur (ou quiconque constitué en autorité agissant pour lui) dès qu'il se rend compte, par lui-même ou par de la délation, que ses employés se syndiquent «dans son dos », il les convoque dans l'atelier, leur enjoint de cesser leur organisation, sinon, il les menace de \& fermer les portes » de son établissement ou de déménager ailleurs; ou il menace ouvertement de renvoi les employés qui feront partie du

(7) Activités légitimes: Recrutement; réunion de fondation; assemblées d'organisation; nomination d'officiers; affichage; paiement des cotisations; affiliation à une centrale; délégué d'atelier; comité de griefs; négociation; délégation à un congrès syndical, etc. 
syndicat, ou il interroge un à un dans son bureau ceux qu'il soupçonne d'avoir été les artisans de l'organisation; ou il leur défend, sous la menace voilée de représailles, de s'affilier à une centrale ouvrière. Et le même employeur fait souvent suivre ses déclarations de quelques congédiements illégaux camouflés sous de faux prétextes, mais qui ont leur effet psychologique sur les employés. Souvent aussi tels congédiements sont effectués quand l'employeur apprend de la C.R.O. que ces employés ont demandé leur certification. Devant cette nouvelle, que ne fait-il pas encore si cela s'est fait à son insu? Il cherchera à les faire démissionner du syndicat même après la certification de celui-ci; il leur fera signer une déclaration qu'ils sont satisfaits de leurs conditions de travail; ou il recourra à toute autre manoeuvre de contestation injustifiée ou à des procédures dilatoires, pour « entraver» l'association de ses employés dans ses activités légitimes vers la conclusion d'une convention collective de travail; ou il refusera absolument de négocier ou, s'il a négocié pour la forme, il refusera d'aller à la conciliation.

Le deuxième paragraphe de l'article 20 peut paraître inutile en ce qui est de la possibilité qu'une « association de salariés » ou qu'un agent d'affaire ou tout autre officier de telle association « adhère " à une association d'employeurs ou «cherche» à en entraver la formation, mais quant à «chercher à dominer les activités 》 d'une association d'employeurs, cela s'est vu de la part de certaines associations ouvrières au comportement assez souvent arrogant, sans cesser de poser en modèle du syndicalisme ouvrier.

Dans le même paragraphe « association de salariés 》 devient, dans le texte anglais de la Loi, « association of employees». L'expression * wage earners », serait mieux que «employees » pour désigner * salariés 》.

\section{CONTRAINTE CONTRE '.E LIBRE CHOIX D'UNE ASSOCIATION}

L'article 21 précise certains actes de l'employeur qui sont de nature à nuire au recrutement d'une association et à ses diverses activités. Les deux infractions les plus fréquemment commises par un employeur sont l'intimidation et le renvoi abusif.

Ce sont là les deux façons d'agir d'un employeur anti-syndical, dont les syndicats ouvriers ont le plus souvent à se plaindre auprès de la C.R.O. Vu la fréquence et la gravité de telles plaintes, notamment pour congédiements illégaux, la Commission, pour l'acceptation de telles requêtes et pour fin d'enquêtes, avait établi des règles de procédure dont la lenteur et l'inefficacité n'avaient d'égal que les déficiences de la Loi. Il est inutile de les rappeler ici, vu les amendements apportés à l'article 21 de la Loi en décembre 1959 par les articles 21a, 21b, 21c, 21d, 21 . 
En regard du deuxième alinéa de l'article 21, relativement au renvoi d'un salarié pour une «cause juste, et suffisante», il est inutile de s'arrêter ici au cas d'indiscipline véritable motivant la suspension temporaire d'un employé, ou sa réduction à un poste inférieur dans l'atelier. Mais il convient de considérer les cas de renvoi d'un employé soit à cause d'incompétence ou d'insuffisance de rendement, soit à cause du ralentissement de la production, soit à cause d'une réorganisation technique de l'entreprise. C'est notamment dans les cas de renvoi de ce genre où la Commission doit s'assurer qu'il y a eu «cause juste et suffisante » c'est-à-dire selon le froit et l'équité et une circonstance suffisamment sérieuse.

Le renvoi d'un employé pour cause de ralentissement de la production ou de réorganisation technique de l'entreprise doit être prouvé par la production de pièces documentaires officielles par l'employeur et par son témoignage oral devant la Commission. Préalablement à cette preuve les inspecteurs de la C.R.O. auront pu être amenés à constater sur les lieux les faits précédemment allégués par l'employeur. Pareille preuve établit ordinairement que le renvoi n'a pas été effectué à cause d'activité syndicale permise par la Loi. Parfois il reste cependant à démontrer que l'employeur n'a pas violé le droit d'ancienneté de l'employé congédié, en ayant gardé à son emploi des employés moins anciens. Cela en supposant que telle règle du droit d'ancienneté existât dans l'entreprise concernée soit par la coutume soit par un contrat de travail.

Le renvoi d'un employé pour incompétence (insuffisance de rendement ou travail mal fait) n'est pas facile à prouver par l'employeur s'il s'agit d'un employé ayant déjà plusieurs années de service dans l'entreprise concernée. En pareil cas il est rare que l'employeur tente de faire sa preuve, car la Commission peut obtenir - ce qui arrive souvent de la part des camarades de travail de l'employé des témoignages contraires aux affirmations de l'employeur.

En définitive, avant les amendements précités à l'article 21, il arrivait qu'en de nombreux cas de renvois injustes l'employeur ne tentait pas de faire la preuve du contraire. Il invoquait multiples manquements à la discipline par l'employé congédié et refusait obstinément de le réengager si telle était la recommandation que lui faisait la Commission. Souvent, aussi, il refusait sous le prétexte de ne pas vouloir «diminuer » l'autorité du contremaître... L'employeur ne pourra plus désormais recourir à de tels prétextes avec les nouveaux articles 21a et suivants de la Loi des relations ouvrières, lesquels articles imposent à l'employeur de réintégrer l'employé congédié illégalement et de l'indemniser pour le salaire perdu de ce fait.

\section{INTIMIDATION ET MENACES}

L'article 22 concerne particulièrement le comportement des organisateurs syndicaux: a) lorsqu'ils entreprennent de syndiquer un établis- 
sement industriel; b) lorsqu'une union cherche à supplanter une association déjà en place; c) lorsque deux syndicats tentent au même moment ou presqu'ensemble de syndiquer chacun de son côté le même groupe de salariés.

L'employeur antipathique est habituellement le premier aussi à alléguer, souvent encore en les exagérant, des actes d'intimidation ou de menaces contre un syndicat qui cherche à supplanter dans son établissement, une «union de boutique» qu'il protège. Si le syndicat libre craint d'être évincé par l'employeur il dénoncera lui-même, s'il y a lieu, les pratiques interdites de l'«union de boutique». Enfin lorsque deux associations rivales libres se font concurrence en même temps pour organiser les ouvriers d'une entreprise ou d'un groupe spécifique de ces derniers, il arrive que l'une accuse l'autre de violation à l'article 22 ou que les deux s'accusent mutuellement de pratiques interdites.

\section{ACTIVITÉS PROHIBÉES}

L'article 23 défend aux salariés de faire du recrutement syndical pendant les heures de travail ni de se réunir au lieu du travail, sauf si l'employeur y consent. Toutefois si ce dernier donne son consentement, sa collaboration sera rarement désintéressée et l'association de ses employés sera rarement libre et indépendante dans ses activités.

Généralement, cependant, les salariés d'une entreprise ne tentent pas de s'organiser eux-mêmes. Ils n'osent en demander la permission à l'employeur. Ils craignent habituellement sa réaction défavorable. Ils souhaitent que quelqu'un de l'extérieur vienne les syndiquer. Or aucun organisateur syndical, généralement, ne demandera non plus la permission à l'employeur. Il sollicitera les employés en dehors des heures de travail, à la sortie de l'atelier ou à leur domicile. Mais il arrivera que parmi les auxiliaires qu'il s'est trouvés dans l'entreprise, quelques-uns, oubliant la consigne ou trop zélés, solliciteront d'autres salariés pendant les heures de travail. Telle infraction, lorsqu'elle est rapportée à l'employeur, coûte très souvent cher à celui qui l'a commise.

C'est une pratique interdite imputable seulement à chaque individu qui l'a commise, mais qui n'empêchera pas l'association, si elle est formée, de présenter une requête en reconnaissance syndicale à la C.R.O.

Telle pratique interdite n'est passible seulement que de la pénalité prévue à l'article 44 de la Loi des relations ouvrières. L'employeur ne recourt habituellement pas à cette sanction, satisfait qu'il est d'avoir renvoyé l'employé en faute.

\section{RESTRICTION DU DROIT DE GRÈvE}

L'article 24, au premier paragraphe, interdit l'exercice du droit de grève ou contre-grève tant qu'une association «n'a pas été reconnue »; 
qu'elle «n'a pas épuisé les procédures voulues pour conclure une convention collective » et qu'il «ne s'est pas écoulé quatorze jours 》 après la décision d'un conseil d'arbitrage.

Voyons içi, le cas d'une sentence arbitrale qui, après le délai de quatorze jours, n’a pas été agréée par l'empolyeur ou a été refusée par lassociation. L'un ou l'autre peut recourir immédiatement à la greve ou à la contre-grève. Mais si l'un ni l'autre n'agit immédiatement, quel délai leur est alloué pour exercer ce droit? La Loi ne prévoit aucun délai. Du côté de l'association ouvrière la Commission a été amenée à poser la règle de lui accorder un délai de trois mois pour exercer son droit à la grève de la date où a été rendue une décision arbitrale.

\section{DÉfENSE DE CHANGer Les CONDITIONS DE TRAVAIL}

L'article 24 stipule aussi que «tant que les conditions» relatives à la reconnaissance et à l'arbitrage, «n'ont pas été remplies, un employeur ne doit pas changer les conditions de travail ${ }^{8}$ de ses salariés sans leur consentement 》. Le sens de cette mise en garde à l'employeur n'est-il pas que dès qu'il est avisé par la Commission que l'association de ses employés a demandé sa certification, qu'il ne peut plus, seul, changer les conditions de travail de ses employés, mais quil a, au contraire, l'obligation d'attendre que l'association soit certifiée pour négocier avec elle une convention de travail et d'attendre même, si nécessaire, jusqu'après les stades de la conciliation et de l'arbitrage?

Mais il n'en est pas toujours ainsi. Il arrive qu'un employeur, au premier stade de la négociation, surtout quand elle traîne en longueur, change les conditions de travail, notamment en ce qui touche le salaire, et ce, "sans le consentement 》 de ses employés. L'employeur, par exemple, qui accorde une augmentation de salaire avant de négocier ou pendant les négociations le fait dans le but de détourner ses employés du syndicat et d'annuler ainsi son efficacité ou de le faire déchoir. Lorsque la Commission est saisie d'une plainte contre telle contravention par un employeur à l'article 24 de la Loi des relations ouvrières il est sommé de se conformer au dit article 24 en rétablissant les conditions telles qu'elles étaient avant la certification de l'association, faute de quoi il est passible de poursuites et des pénalités prévues par la loi. Si tel employeur ne s'amende pas, l'association certifiée n'est plus intéressée à poursuivre les négociations avec un employeur visiblement de mauvaise foi et demande habituellement à la C.R.O. de prier le Procureur général

(8) L'expression \& conditions de travail » dans la Loi des relations ouvrières (Art. 24) devient « conditions of employment dans le texte anglais de la Loi; la même traduction anglaise se répète dans la Loi des Différends entre les Services publics et leurs salariés (article 2.f version francaise); mais nous trouvons \& working conditions \$ dans la Loi de la convention collective (article 1-d). D'autre part, dans la Loi des syndicats professionnels l'expression \& conditions de travail ? se lit (art. 6-9o) conditions collectives de travail \$. 
de le poursuivre en justice ${ }^{9}$. Ce dernier $y$ acquiesce quand il considère l'intérêt général compromis.

Un employeur viole encore l'article 24 de la L.R.O. si après la date d'expiration d'une convention collective il a changé les conditions de travail de ses salariés sans leur consentement, que cela soit avant ou pendant les négociations du renouvellement de la convention.

La stipulation que l'employeur ne peut changer les conditions de travail de ses salariés sans leur consentement, a-t-elle un sens équivoque? S'agit-il du consentement de chacun des salariés ou de leur consentement collectif? Des procureurs soutiennent qu'il suffit du consentement individuel des salariés tant que leur association n'est pas certifiée; d'autres prétendent qu'il faut obtenir leur consentement collectif en tant que membres d'une association en instance devant la C.R.O. pour sa reconnaissance légale.

Par ailleurs nombre d'associations s'abstiennent de faire la grève préférant tenter d'entamer des négociations post-arbitrales. A cette fin elles demandent l'aide d'un conciliateur du ministère du Travail. Bien que cette procédure ne soit pas prévue par la L.R.O., elle s'est instituée graduellement avec l'agrément du ministre du Travail et en quelque sorte sous l'empire de certaines dispositions de la Loi des différends ouvriers.

La négociation post-arbitrale s'est révélée une procédure généralement fructueuse. Mais elle est parfois infructueuse et suivie de la grève que l'on avait voulu éviter. En pareil cas la grève est-elle légale? Il est permis d'en douter.

\section{RALENTISSEMENT de LA PRODUCTION}

Une autre pratique interdite est le ralentissement de la production, suivant l'article 25.

Le ralentissement de la production dans une entreprise peut être le fait d'une association ouvrière qui recourt à ce moyen pour hâter la conclusion d'une convention collective dont la négociation traîne en longueur; ou il peut être attribuable au refus d'une association de permettre à ses membres, sous peine d'amende, de travailler du temps supplémentaire, même lorsque c'est une obligation bien qu'un peu mitigée, dans la convention collective ${ }^{10}$.

(9) Par l'amendement à l'article 49, la Commission peut dorénavant poursuivre elle-même.

(10) Voir dans Op. cité, p. 255, une décision de la C.R.O. dans Canadian Lithographers Association vs Amalgamated Lithographers of America, local 27. 


\section{Changement d'AfFiliation}

En vertu de l'article 26 il n'est pas permis à une association de s'affilier à une autre association pendant la durée d'une convention, sauf dans les soixante jours précédant la date d'expiration de la convention.

Le sens de l'article 26 est bien qu'une association liée par une convention collective doit attendre au délai prescrit avant la fin de la convention pour tenter, si elle le désire, des démarches en vue de s'affilier à une autre association. "S'affilier à une autre association 》 peut avoir ici deux sens: 1o.-s'affilier (ou se fusionner) à une autre association dans la même entreprise, dans une unité de négociation agrandie, par exemple; 20.-s'affilier à une association centrale autre que celle à laquelle elle est déjà affiliée. Car ce n’est qu'ainsi, par un changement d'allégeance, qu'une association certifiée peut «devenir membre » collectif d'une autre association extérieure.

L'article 26 défend aussi à un "groupe de membres » d'une association liée par contrat de s'affilier à une autre association ou d'en devenir membre, c'est-à-dire de se détacher de l'association certifiée pour s'intégrer à une autre sauf dans le délai prescrit avant la fin de la convention.

Cette interprétation de l'article 26 par la C.R.O. fait que, selon elle, le même article n'empêche aucun employé membre d'une association certifiée de faire en tout temps de la propagande ou sollicitation individuelle privément en faveur d'une autre association et ne défend pas non plus à aucun employé (sans cesser d'être membre de l'association certifiée) de devenir membre d'une autre association qui a le dessein de se faire reconnaître à la place de l'association actuelle selon les exigences de la Loi des relations ouvrières.

Lorsque, conformément à l'article 26 , une association veut s'affilier à une autre association dans le délai prescrit, elle doit en informer la C.R.O. afin de changer le nom de l'association sur le certificat de reconnaissance. Mais, au préalable, la Commission doit s'assurer si le changement d'affiliation a été consenti par la majorité des membres de lassociation, non pas seulement de ceux présents à l'assemblée où la décision a pu être prise. Elle enquête pour savoir s'il y a une résolution de voter en ce sens en assemblée plénière, après convocation spéciale à cette fin, et le nombre de membres présents. Puis elle procède au sondage d'un certain nombre de membres pour avoir la confirmation des faits précités.

\section{Devoirs des associotions}

Toute association qui demande à être reconnue doit, selon l'article 27 , présenter avec sa requête copie de sa constitution et règlement et autres informations relatives au droit d'entrée etc. 
La production des documents précités permet à la C.R.O. de connaître le but, la nature des activités et le mode d'administration de l'association requérante pour en établir l'identité à savoir, si elle est une association autonome, ou subordonnée, ou affiliée à une centrale, de même que pour en connaître la bonne foi.

En regard de l'alinéa b) de l'article 27, relatif aux droits d'entrée et aux cotisations, la C.R.O., avant 1946, devait se rapporter aux statuts et règlements de chaque association requérante pour connaître les conditions établissant la qualité de membre en règle, pour déterminer ensuite le caractère représentatif de l'association. Etant donné la grande diversité des constitutions syndicales soumises à la C.R.O., cette dernière vit bientôt la nécessité d'arrêter des critères communs propre à faciliter l'émission de certificats de reconnaissance syndicale à toutes les associations requérantes à cet effet. Or, à cette fin, elle décida en 1946 d'adopter son Règlement no 1.

Ce qui est stipulé à l'alinéa b de l'article 27 fait maintenant l'objet du Règlement no 1 aux prescriptions duquel doit se conformer toute association requérante.

L'alinéa b) contient une équivoque. Elle consiste dans l'expression \& droit d'initiation, d'entrée »... « D'entrée » étant séparé d'< initiation * par une virgule, le sens apparent est que le substantif «droits » mis au pluriel précède aussi « entrée». Ce qui ferait croire qu'une association de salariés peut demander à ses membres un droit d'initiation et un droit d'entree. Ce qui n'est pas conforme à la coutume syndicale qui n'exige pas un droit d'initiation distincte du droit d'entrée.

Très rares sont les associations certifiées qui pensent à transmettre a la C.R.O. les noms et adresses de leurs officiers après chaque élection. La non observance des associations en général à cette stipulation de l'article 28 est cause qu'en maintes occasions les communications de la C.R.O. n'atteignent pas en temps voulu les officiers auxquels sont destinées quand il y a eu changement de titulaires.

\section{Lo Commission de relations ouvrières}

\section{Pouvoms admintstratifs ET JUdictames}

Concernant la Commission de relations ouvrières mes observations ne porteront que sur le double caractère de ses séances et sur l'exercice de ses pouvoirs tels que stipulés aux articles $38,39,41$, 4la et 41 b.

Les pouvoirs de la C.R.O. sont de deux ordres: administratif et judiciaire. De même ses séances ont un caractère administratif et un caractère judiciaire ${ }^{11}$. Ses décisions, à mon sens, sont judiciaires lors-

(11) Voir Handbook on Canadian Labour Law par Alfred Cosby Chrysler 206 et 285-286. Editeur, The Carswell Co., Toronto. 
qu'elles disposent d'affaires relatives à l'émission, à l'amendement, au refus ou à la révocation d'un certificat de reconnaissance syndicale; elles sont d'ordre administratif lorsqu'elles disposent d'affaires se rapportant à l'unité de négociation, au dépôt de convention collective, aux procédures de conciliation et d'arbitrage et enfin à toute affaire se rapportant à sa régie interne et à son service d'inspection. Mais lorsqu'en séance privée la Commission délibère sur des litiges de droit entre des parties qui ont comparu devant elle en séance d'audition, elle fait alors fonction de tribunal judiciaire.

De même lorsqu'elle accorde une autorisation de poursuivre, sur demande d'une partie lésée.

D'autre part, la Commission prend réellement figure de tribunal jjudiciaire lorsqu'elle siège en séance d'audition pour entendre l'interrogatoire de témoins par les avocats des parties contestantes dans tout litige appelé à s'instruire devant elle.

La conduite de ses séances d'audition s'inspire très peu du code de procédure civile ${ }^{12}$ lorsque le procureur de l'association ouvrière n'est pas avocat.

Ce qu'il importe tout particulièrement de souligner ici c'est que lorsque la preuve à faire devant la C.R.O. oblige un salarié à témoigner contre son employeur en sa présence ou en présence de ses représentants, ce témoin sent le poids de son infériorité, de son état de mineur devant son employeur et lorsqu'il est soumis à un interrogatoire il se sent comme mis au pilori. Ses réponses lui attireront-elles des représailles de la part de l'employeur ou de ses représentants à brève ou à longue échéance? Il met en jeu sa position ou son avancement. Situation plus que pénible, douloureuse même quand elle touche un chef de famille. Il devra souvent être héroïque pour dire la vérité.

Souvent, avant d'entendre tel témoin, le procureur syndical demandera à la Commission de lui garantir la protection de la L.R.O. contre toute représaille ou discrimination. Malheureusement l'expérience enseigne que la protection assurée au témoin par le président de la C.R.O. en un tel moment demeure souvent une simple déclaration platonique. Car quelques semaines ou quelques mois plus tard, sous un prétexte quelconque, des représailles seront souvent exercées contre ledit témoin.

\section{ExERCICE dES POUVOIRS DE LA C.R.O.}

Par l'article 38 la Commission peut faire des règlements pour l'exercice de ses pouvoirs, de sa régie interne et la «mise à exécution de la Loi ». L'article 38 contient deux points importants à être soulignés: «l'exercice des pouvoirs de la Commission» et la «conduite de ses

(12) Voir Op. cit., p. 62. 
séances ». Deux autres points mériteraient de l'être aussi: la « procédure de ses enquêtes » et la «forme des rapports » à lui adresser. Mais il est inutile de revenir sur le premier de ces deux derniers points car il a été en grande partie exposé dans la première tranche de cette étude et qu'il en sera aussi question dans la troisième et dernière tranche, où il sera également traité du second point.

«L'exercice des pouvoirs » de la Commission est la mise en application de la Loi des R.O. et l'interprétation qu'elle en fait. Les «pouvoirs » comme aussi les devoirs de la C.R.O. sont particulièrement stipulés à la Section VI de la L.R.O. Les pouvoirs et devoirs de la Commission sont contenus dans son Règlement no 1 , de même que dans les règles de jurisprudence qu'elle a élaborées au cours des années. Dans l'examen des prochains articles de la Loi il sera montré que certains pouvoirs de la C.R.O. sont contestés, méprisés peut-être même par certains procureurs et magistrats. L'interprétation habituelle que fait la Commission de certaines dispositions imprécises ou équivoques de la L.R.O. prend aussi forme de jurisprudence, pas toujours écrite, pour les cas similaires qu'elle a à décider.

Par ailleurs, que de fois la Commission est appelée à décider dans le silence de la Loi. Est-ce alors son pouvoir discrétionnaire de se prononcer qui entre en jeu ou est-ce l'obligation morale de régler l'affaire qui l'occupe si elle est dans le cadre de ses attributions selon l'esprit de la L.R.O.? En telle occurrence elle prend le risque de décider dans un sens ou dans l'autre. Telle décision vaut tant qu'elle n'est pas contestée ni renversée par un jugement de cour.

\section{POUVOIR DISCRÉTIONNAIRE}

De tous les articles de la Loi qui confèrent à la C.R.O. ses pouvoirs, l'article 39 est celui qui ajoute le plus au pouvoir discrétionnaire que lui donne l'économie de la Loi. Cest là une disposition précieuse qui permet à la Commission de décider dans «l'absence d'une disposition règlementaire applicable à un cas particulier $», \ldots$ de «prescrire tout acte ou formalité » à être observé.

Un cas fréquent où la Commission s'autorise de l'article 39 c'est lorsqu'elle juge nécessaire dans certain litige d'ordonner un vote pour connaître la volonté des salariés dans une entreprise, à savoir s'ils désirent être représentés par une association ou non ou s'ils préfèrent adhérer à une association plutôt qu'à une autre.

Il est trois autres circonstances où la Commission ordonne la tenue d'un vote en vertu du même article: 10.-Quand les parties en litige, pour s'éviter l'ennuyeuse procédure de faire preuve et contre-preuve devant la Commission, s'entendent pour lui demander par écrit de vider leur litige par un vote parmi les salariés; 20.-Quand sur demande d'une partie interressée ou que d'office la Commission décide de faire voter 
les salariés sur le choix à faire par eux-mêmes de l'unité de négociation, c'est-à-dire sur la composition physique de l'unité par l'inclusion ou non de certaines catégories de salariés; 3o--Quand appelée à reviser l'effectif de l'association reconnue avant le renouvellement de sa convention collective avec un employeur, la Commission décide de faire cette revision au moyen d'un vote-enquête.

D'aucuns prétendent que le résultat d'un vote, tenu en vertu de l'article 39 et même de l'article 8 , touchant la contrainte doit être suivi d'une enquête afin de constater si les prescriptions du Règlement no 1 sont remplies pour posséder pleinement le caractère représentatif. La Loi laisse présumer que l'association verra à recruter le nombre de membres requis pour correspondre à la majorité des votes obtenus en sa faveur.

POUVOIR DE REVISION OU RÉVOCATION

Le non des moindres pouvoirs conférés à la C.R.O. par l'article 41 est celui de « reviser ou révoquer pour cause toute décision et tout ordre rendus par elle et tout certificat qu'elle a émis ».

Toutefois la rigueur de ce pouvoir est tempéré par l'article $41 \mathrm{~b}$ qui oblige la C.R.O. à « entendre » les parties, si elles le désirent, avant d'exercer ce droit s'il y a lieu.

Trois causes principales peuvent obliger la C.R.O. de reviser ou révoquer une décision ou un ordre rendus par elle, à savoir: une erreur dans l'énoncé d'une décision, des faits nouveaux portés à sa connaissance, une infraction à la L.R.O. par l'une des parties devant elle.

C'est après l'émission d'un certificat de reconnaissance que la Commission est appelée ordinairement à reviser une décision, soit pour corriger une erreur dans la désignation des parties concernées dans ledit certificat, soit pour y rectifier une erreur dans le libellé de l'unité de négociation, soit en regard du caractère représentatif de l'unité de négociation.

Le caractère représentatif de l'unité de négociation est le plus important point à souligner, car, ici, la revision a pour but de constater si l'association certifiée a conservé la majorité de l'effectif des salariés concernés, particulièrement à l'époque du renouvellement de la convention collective.

Faute d'aucune stipulation précise dans la L.R.O. sur le droit de reviser l'effectif syndical certifié ni quant à la date où ce droit peut être exercé, l'article 41 est invoqué habituellement par nombre d'employeurs pour demander à la C.R.O. de procéder à telle revision à l'époque où se termine une convention collective, avant d'entamer des négociations pour son renouvellement, l'employeur voulant alors s'assurer que l'association reconnue continue de représenter la majorité de ses employés. 
La Commission se rendit vite à l'évidence de la nécessité pour l'association certifiée de maintenir son caractère représentatif pour préserver son droit d'agent négociateur. Seulement la Commission a jugé nécessaire d'établir certaines règles à être observées avant d'accepter une demande de revision de l'effectif syndical reconnu.

La première règle, elle l'a tirée de l'article 15 à l'effet de n'accepter une demande de revision d'effectif d'une unité certifiée que dans le délai du $60 \mathrm{e}$ au $30 \mathrm{e}$ jour précédant l'expiration d'une convention. La Commission fera également la revision si la demande, dans le même délai, provient d'un groupe majoritaire d'employés démissionnaires dans l'unité certifiée, soit l'association.

Si assez longtemps (habituellement un an) après l'émission d'un certificat de reconnaissance, il n'a pas été conclue de convention collective, la Commission acquiescera aussi à une demande de revision de la part de l'employeur.

En tout autre circonstance, après l'émission d'un certificat de reconnaissance, la Commission refuse de reviser l'effectif de l'unité de négociation tant que les trois stades prévus par la L.R.O. n'ont pas été épuisés à savoir la négociation, la conciliation et l'arbitrage. Même après le prononcé d'une sentence arbitrale, la Commission laisse écouler un délai de trois mois avant de reviser l'effectif certifié pour permettre aux parties, s'il y a lieu, la signature d'une convention collective ou l'exercice de sanctions économiques de part et d'autre.

Il arrive aussi que peu après la certification d'une association, un employeur allègue le retrait de la majorité de ses membres et demande la revision de son caractère représentatif . La Commission refuse catégoriquement la revision, l'expérience lui ayant appris que les démissions en pareil cas résultent presque toujours d'une pression indue exercée par l'employeur.

Jamais non plus la Commission ne revise un effectif certifié pendant le cours d'une convention ni lorsqu'une convention a été renouvelée automatiquement. Elle ne fait pas telle revision non plus pendant une grève ou contre-grève. La revision de l'effectif de l'unité de négociation peut se faire de deux manières: soit par l'examen des livres de cotisations de l'association reconnue soit par un vote enquête. La procédure régulière est la première. La seconde est exceptionnelle. La Commission y recourt, en vertu des pouvoirs que lui confère l'article 39 , dans des cas particuliers où la longue durée d'un litige justifie la Commission par équité pour l'association certifiée et pour bien connaître la volonté des salariés, d'ordonner un vote-enquête parmi ces derniers en guise de revision de l'effectif.

\section{DÉCISION SANS APPEL}

En 1953, à la demande des unions ouvrières l'article 4la fut ajouté à la L.R.O., en vue d'enrayer l'abus des brefs de prérogative pris par 
maints employeurs contre les décisions de la C.R.O. Les enquêtes et les procédures judiciaires qui s'ensuivaient pendant un temps interminable, étaient préjudiciables aux syndicats ouvriers.

L'adoption de l'article 4la n'a pas diminué les recours devant les tribunaux civils pour reviser les décisions de la C.R.O. Au contraire les injonctions prises contre la Commission ont continué d'augmenter. L'article 4la a paru être interprété par les avocats et les tribunaux comme un défi contre eux. Les brefs de prohibition et d'injonction sont les plus fréquemment pris contre la C.R.O.

Après l'entrée en vigueur de l'article 4la les premiers jugements des tribunaux supérieurs sur les appels logés contre la Commission revendiquèrent le droit des mêmes tribunaux d'entendre les appels contre la C.R.O. quand ses décisions excèdent sa juridiction.

Depuis 1944 (date de formation de la C.R.O.) jusqu'à 1952, onze brefs en annulation de décisions ont été pris contre la Commission; de 1953 à 1958 il y en a eu 15 . Soit 15 brefs en annulation de décisions dans la deuxième période de cinq années contre 11 dans la première période de 8 ans. Et le rythme de tels brefs n'a pas diminué depuis 1958.

\section{LE CODE DE PROCÉDURE CIVILE}

L'alinéa «c» de l'article $41 \mathrm{a}$, relatif à l'article 50 du code de procédure civile est devenu conséquemment une disposition qui n'a plus de sens, car l'expérience démontre que les dispositions de l'article 50 du Code de procédure civile s'applique à la C.R.O. Au juste, que dit l'article 50 du Code de procédure civile? Ceci:

- A l'exception de la Cour du banc du roi, les tribunaux relevant de la législature de Québec, ainsi que les corps politiques et les corporations dans la province, sont soumis au droit de surveillance et de réforme de la Cour Supérieure, en la manière et dans la forme prescrites par la Loi, sauf dans les matières que la loi déclare être du ressort exclusif de ces tribunaux, ou de l'un quelconque de ceux-ci, et sauf dans les cas où la juridiction découlant du présent article est exclue par quelque disposition d'une loi générale ou particulière. > (5-6 SRQ. 11, ch. 15)

Indiscutablement, à l'exemple de tous les tribunaux inférieurs, la C.R.O., corps à la fois administratif et judiciaire, est soumise au «contrôle de la Cour Supérieure» lorsqu'elle excède sa juridiction. Or, sauf exception, tout juge à qui la demande en est faite, accorde très facilement tout bref de prérogative demandé contre la C.R.O. $\mathrm{Ne}$ devrait-il pas au préalable s'assurer du bien fondé de telle demande eu égard aux pouvoirs conférés à la Commission par la Loi des relations ouvrières et le Règlement no 1 de la C.R.O.? Presque toujours cette responsabilité est laissée au tribunal qui doit en décider. Lorsqu'il 
s'agit notamment de brefs de prohibition ou d'injonction avec ordre de surseoir c'est le tribunal qui en décide et cela, six mois, un an ou deux ans après. Les préjudices qui s'ensuivent lèsent beaucoup plus lourdement le syndicat et les employés que l'employeur.

Depuis cinq ou six ans, suivant l'exemple des employeurs, nombre de syndicats ouvriers ont aussi pris des brefs d'injonction ou de prohibition contre certaines décisions de la C.R.O. Les motifs généralement allégués par les syndicats sont, soit un excès de juridiction, soit une soit-disant fausse interprétation de la L.R.O., soit l'inconstitutionalité d'un article de la Loi. Dans ce dernier cas il s'agit habituellement de larticle 24 concernant la restriction du droit de grève.

\section{De la grève illégale et sa responsabilité}

L'article 43 stipule les pénalités à être imposées aux auteurs d'une grève illégale. Examinons d'abord les divers aspects d'une grève illégale, et qui en porte la responsabilité.

Il y a la grève préméditée par un syndicat par voie de résolution autorisant l'exécutif de la déclarer en temps opportun, sans égard aux prescriptions de la Loi; il y a la grève fomentée par les officiers d'une union ou par un groupe d'ouvriers dans une entreprise, encore à l'encontre des prescriptions de la Loi.

La grève préméditée est, avant son exécution, à la fois un avertissement et une menace; la grève fomentée par les officiers d'un syndicat, est une attaque éclair; fomentée par les ouvriers d'une usine, ça peut être une grève sauvage si c'est contre les directives du syndicat, ça peut être une grève spontanée si les ouvriers ne sont pas syndiqués.

Mais en prévoyant le cas d'une grève provoquée le législateur n'at-il pas pensé que la provocation pouvait être aussi le fait de l'employeur? Que cela soit venu ou non à l'esprit du législateur, la chose se produit dans le monde du travail. Or lorsqu'une grève illégale est due à la provocation d'un employeur, pourquoi l'article 43 prescrit-il de pénaliser seulement les grévistes ou leur syndicat et ignore l'offense du provocateur, l'employeur?

En droit naturel n'incombe-t-il pas ici une responsabilité morale à l'employeur, et les grévistes, dans l'illégalité forcée, n'ont-ils pas droit à une exemption ou un adoucissement de la sanction qu'impose la Loi? La Commission s'est inspirée pour la première fois, de cette préoccupation dans la décision qu'elle a rendue le 10 mars 1959 dans l'affaire de la Cie Kendall Bros. vs le Syndicat national des Camionneurs de Montréal ${ }^{13}$. 
En cette affaire la Commission a rejeté la requête de l'employeur demandant la décertification du syndicat pour grève illégale, pour les deux motifs suivants: à savoir

« que la preuve a révélé que le patron porte une grande responsabilité de l'arrêt du travail par sa négligence d'installer des chaufferettes dans ses camions (et que) cette négligence est confirmée par le fait qu'il a immédiatement posé des chaufferettes et qu'il n'a pas congédié les chauffeurs concernés. ?

\section{INTERVENTION SINGULIÈRE DE LA COMMISSION}

Que fait la C.R.O. quand elle est saisie d'une plainte ou d'une accusation de grève illégale?

La Commission fait une mise en demeure conditionnelle à l'association contrevenante à l'effet que si l'accusation est fondée, d'avoir à se conformer à la loi, sinon, que la loi suivra son cours.

Ordinairement, à moins qu'un arrangement intervienne entre l'employeur et l'association, une audition est ordonnée d'office par la Commission ou à la demande de la partie plaignante. Après preuve faite la Commission fait le prononcé de lillégalité de la grève pour décider de la sanction à être imposée. Mutatis mutandis la même procéduce est suivie à l'occasion d'une contre-grève illégale.

Immanquablement la coutume existe que l'employeur lésé par une grève illégale demande de révoquer le certificat de reconnaissance syndicale à l'association en faute. Cela est naturellement le geste de l'employeur qui ne veut pas d'union ouvrière dans son établissement, ou qui en préfère une de son choix, ou qui, à juste titre, veut se libérer d'une association qui lui a fait subir non seulement une grève illégale, mais, à la fois, injuste. Peu importe le cas, selon aussi une vieille habitude, la Commission acquiesce à la demande de l'employeur et révoque le certificat de reconnaissance.

Ignorant, tout comme l'employeur, les articles 43,46 et 47 de la L.R.O. prescrivant des pénalités contre les auteurs d'une grève illégale, la Commission s'en est constamment rapportée majoritairement, ${ }^{14}$ à l'article 41 qui l'autorise, "pour cause», de révoquer un certificat de reconnaissance syndicale. Les décisions majoritaires à cet effet s'appuient sur l'argument qu'il est justifié de retirer le certificat de reconnaissance syndicale pour grève illégale, afin de libérer l'employeur de l'obligation de négocier avec une association qui a ainsi violé la Loi.

De leur côté les commissaires dissidents soutiennent qu'en pareille occurrence la procédure normale serait, après avoir prononcé l'illégalité

(14) Jusqu'au début de 1960 - depuis l'auteur a cessé d'être commissaire. 
d'une grève, d'indiquer à l'employeur lésé les pénalités prescrites par la Loi (articles 43-44-46-47) qu'il peut exiger contre l'union coupable de grève illégale. En décertificant le syndicat, la Commission, prétendentils, rend une décision punitive de nature contraire à ce que prescrit la L.R.O. A l'argument qu'il convient de libérer l'employeur de l'obligation de négocier, ils opposent que cette obligation se trouverait suspendue pour un temps par le fait des procédures judiciaires que P'employeur prendrait contre l'union pour la soumettre aux pénalités de la Loi et que ce n'est qu'après que l'union s'en serait acquittée que l'employeur redeviendrait obligé de négocier avec elle. Selon eux, quatre causes principalement, en vertu de l'article 41 , justifient de décertifier une association pour grève illégale:

1-Quand, par une décision syndicale, elle agit avec une mauvaise foi calculée dans le mépris des lois et de l'autorité;

2-Quand elle est dirigée par un ou plusieurs officiers ou organisateurs communistes, (article 6);

3 - Quand elle groupe des salariés employés dans un service public au sens de la Loi des Différends entre les services publics et leurs salariés;

4 Quand elle ordonne ou permet à un groupe de ses membres liés par une convention collective, de rompre ladite convention par une grève de sympathie en faveur d'autres employés de la même entreprise.

5 -Quand elle recourt à toute forme de boycottage par ses membres contre un employeur pour forcer ses employés à changer d'allégeance syndicale.

Hors les cinq circonstances précitées tout autre cas de grève illégale, particulièrement celle provoquée par un acte arbitraire de l'employeur ne devrait être l'objet que de pénalités.

Si par sa culpabilité une association a mérité la décertification, les travailleurs qui ont participé à la grève dans l'entreprise concernée, s'ils retournent au travail pour le mếme employeur (en supposant qu'ils n'ont pas été remplacés) devraient cependant avoir le droit, en tant que personnes libres et collaboratrices dans l'entreprise, de s'associer dans une nouvelle association n'ayant rien de commun avec la précédente et ensuite de la faire reconnaître par la C.R.O. Ou, pour le moins, faute de certification, la nouvelle association devrait pouvoir négocier une convention collective provisoire d'un an. En ce faisant les parties ne négocieraient que de gré à gré sans les recours prévus par la Loi. A l'expiration d'une telle convention l'association recouvrirait son droit à la reconnaissance syndicale.

Causes de la grève illégale

En regard de la L.R.O., il y a sept circonstances où peut éclater ordinairement une grève illégale, à savoir: 
a) pendant la période de formation d'un syndicat;

b) pendant qu'il est en insistance devant la C.R.O. pour obtenir un certificat de reconnaissance;

c) après avoir été reconnu par la C.R.O.;

d) pendant l'un ou l'autre des trois stades de la négociation, de la conciliation et de l'arbitrage;

e) avant la fin du délai légal après la sentence arbitrale;

f) après des négociations post-arbitrales;

g) pendant la durée d'une convention.

La grève illégale qui éclate pendant les circonstances, a) b) c) est ordinairement provoquée par des renvois abusifs illégaux effectués par l'employeur. C'est généralement la grève spontanée, soudaine. Par contre la grève qui survient pendant les circonstances d) e) f) g) est habituellement plutôt concertée, sauf que dans ces trois cas elle puisse être encore provoquée par un acte arbitraire de l'employeur.

Aux trois circonstances a) b) c) où une grève est provoquée par la conduite injuste ou illégale de l'employeur, il s'en ajoute une autre: le refus d'un employeur de négocier avec une association que vient de reconnaitre la C.R.O. Refus parfois brutal de rencontrer les représentants de l'association ou tentatives diverses pour se dérober à la Loi. Un employeur peut encore provoquer une grève illégale en violant une convention de travail.

On a représenté qu'interrompre le travail pour tenir une assemblée n'était pas assimilable à une grève. Il reste à déterminer où et quand cette assemblée a été décidée? Pour quel motif? Si cette interruption de travail survient pendant l'un des trois stades de la négociation, conciliation et arbitrage d'une convention collective, la C.R.O. se considère en face d'un fait de grève. Car si celle-ci n'a pas été décidée à une réunion préalable du syndicat, elle est ordinairement plus ou moins concertée, peut-être dans un mouvement de promptitude, lorsqu'elle éclate.

Des employés qui démissionnent collectivement d'une entreprise à loccasion d'un différend, posent-ils un acte de grève? La C.R.O. le croit, s'il y a eu concertation préalable.

Le piquetage sans déclaration de grève, est-ce la grève? La L.R.O. ne contient pas un mot sur le piquetage. C'est un moyen de défense syndicale non prévu par la Loi.

\section{AMende et Autre Peine}

«A moins qu'une autre peine ne soit applicable », stipule l'article 44, «quiconque - commet une infraction» à la Loi - est passible d'une amende de... 
* A moins qu'une autre peine ne soit applicable», cela veut-il dire: soit en vertu de certaines dispositions du Code civil soit en vertu du Code criminel? Dans de tels cas ne s'appliquerait pas l'amende prévue à l'article 44?

Ou doit-on supposer que la révocation d'un certificat de reconnaissance syndicale par suite de grève illégale est en soi une peine imposée au syndicat contrevenant qui le soustrait à l'amende prévue à l'article 44 ?

D'aucuns prétendent par contre que la révocation d'un certificat pour grève illégale - comme le fait habituellement la C.R.O. en se reposant sur l'article 41 - ne soustrait pas le syndicat à l'imposition d'une pénalité si la poursuite l'exige, comme l'autorise l'article 43. Avec force argument Alfred Cosby Chrysler soutient exactement le contraire dans son ouvrage Handbook on Canadian Labour Law, publié en 1957. Voici ce que dit cet auteur des prescriptions de notre Loi en la matière:

\begin{abstract}
\& Under the Quebec Legislation express provision is made for the punishment of every person participating in a violation of any of its terms. And it is a basic rule that where an Act creates an offence and provides a penalty for it, the latter, in the absence of language indicating a contrary intent, is to be presumed to be the only punishment intended. There was nothing from which the slightest implication could be drawn that other punishment was intended, but Quebec board, where employees had gone on strike contrary to law, had imposed other punishment, in the form of cancelling the bargaining agents' certificate of representation compared with which the pecuniary penalties authorized, through substantial, were insignificant. Moreover, the task of the Quebec board, which was concerned with ascertaining whether a certain state of facts was present and, once those facts were found to exist, with recognizing bargaining agents and issuing certificates to them, was incompatible with authority to revoke solely on the ground that there had been a violation of a penal provision of the statuts,, 15
\end{abstract}

Autre témoignage concordant avec le précédent: en 1948, en accordant une injonction interlocutaire pour empêcher le piquetage pendant la grève illégale, le juge Campbell de la Cour Supérieure disait que les articles touchant les pénalités (43 et 44 ) de la Loi des relations ouvrières « sont des articles ancillaires à l'idée générale de la Loi des relations ouvrières qui tombe sous le coup de larticle 92 de l'A.A.B.N. concernant la propriété et le droit civil», et il continuait: «l'article 43 de la L.R.O. ne rend pas la grève illégale, mais il ordonne des pénalités lorsqu'une grève est déclarée avant que certaines conditions ne soient

(15) Handbook Canadian Labour Law, p. 245, The Carswell Co. Ltd., Toronto. 
remplies ». ${ }^{16}$ - Ledit article n'ordonne donc pas la révocation d'un certificat dans un cas de grève illégale. La Commission n'a qu'à prononcer alors l'illégalité de la grève après avoir fait son enquête.

Passibles de pénalités par le fait d'une telle décision, les auteurs de la grève illégale devraient ensuite, me semble-t-il, subir la suspension de leur droit de négociation aussi longtemps qu'ils n'ont pas versé les pénalités exigées par la Loi.

\section{Observations diverses}

\section{DRort DE POURSUTTE À L'ASSOCLATION \& BONA FIDE ?}

L'article 48 stipule que le recouvrement des pénalités prévues par la présente loi doit se faire sur poursuite sommaire suivant la Loi des convictions sommaires de Québec (Chap. 29).

Les associations incorporées en vertu de la Loi des Syndicats professionnels ne présentent pas de difficultés pour être poursuivies, sil y a lieu, suivant la Loi des convictions sommaires de Québec (chap. 29).

Jusqu'à la dernière législature provinciale (1959-60) les associations non incorporées en vertu de la Loi des syndicats professionnels ne pouvaient poursuivre mais pouvaient être poursuivies en vertu de la \&Loi concernant certaines matières spéciales relatives à la Procédure \ (S.R.Q. 1941, chap. 342 , art. 28-29).

Mais voici qu'une nouvelle législation vient d'amender le Code de Procédure civile par les articles $81 \mathrm{a}$ et $81 \mathrm{~b}$. L'article 81a, assujettit l'association «bona fide» à la même procédure judiciaire susdite; l'article $81 \mathrm{~b}$, donne à l'association non incorporée le droit d' « ester en justice \$ pourvu qu'elle obtienne de la Commission un certificat \&attestant 》 qu'elle est « bona fide au sens de la Loi des relations ouvrières \$. La Loi ne mentionne pas la procédure que devra suivre la C.R.O. pour établir cette bonne foi. Le règlement no 1 de la C.R.O. pourra cependant servir de base.

\section{DisSOLUTION D'ASSOCIATION}

En vertu de l'article 50 la dissolution d'une association que peut prononcer la C.R.O. vise ordinairement une association dominée par un employeur dans sa formation et ses activités et qui, ainsi, au surplus, participe avec l'employeur à entraver la formation d'une association libre.

(16) Aird \& Son Limited vs Local 500 International Union of Shoe and Leather Workers of United States and Canada et al. and Association of Shoe Manufacturers of Quebec, 1948; 3 D.L.R. 114; C.S. Quebec 16-12-47; Juge Campbell; C.L.L.R. 31,081 No. 35,083. Reproduit aussi dans: Bulletin des Relations Industrielles, juin 1950 , p. 88 . 
La dissolution d'une association incorporée en vertu de la Loi des Syndicats professionnels est une dissolution qui a un sens légal absolu: elle prend effet par le retrait de l'incorporation effectuée par le Secrétaire de la province, après avoir été avisé à cette fin par la C.R.O. Et cette dissolution en vertu de la Loi des Syndicats professionnels entraîne la liquidation des biens de l'association inculpée.

S'il s'agit simplement d'une association « bona fide », quel sens alors donner à une dissolution prononcée par la C.R.O.? Cela peut avoir deux effets seulement: celui du retrait du certificat de reconnaissance s'il y en a eu un d'émis, ou celui du refus d'en accorder un en rejetant la requête à cette fin. La Commission n'a aucun moyen de vérifier si lassociation concernée s'est effectivement dissoute par voie de résolution et comment s'est fait le partage de ses biens ou si elle continue d'exister clandestinement. ${ }^{17}$ Et aucun avis de sa dissolution n'est publié dans la Gazette officielle du Québec ainsi que cela est exigé dans le cas d'un syndicat incorporé.

Il est évident qu'ordonner la dissolution d'une association est en soi une peine. Mais l'article 50 dit que cela peut se faire \&sans préjudice de toute autre peine». Cela réfère-t-il aux peines prévues aux articles 44,46 et 47 de la Loi des relations ouvrières? Ne serait-ce pas dépasser la mesure?

\section{DÉSUÉTUDE DE L'ARTICLE 53}

Deux dispositions transitoires de la Loi qui n'ont plus aucun sens sont celles de l'article 53.

L'article 53 demande que les conventions collectives existant lors de l'entrée en vigueur de la Loi «soient modifiées de façon à les rendre conformes à l'article 15 » relativement à leur durée.

Le premier alinéa de l'article 53 est à biffer parce qu'il est, depuis longtemps, devenu désuet, vu qu'il n'existe plus de conventions collectives remontant à 1944 qui n'aient été, depuis, modifiées conformément aux dispositions de l'article 15. Pour la même raison est à rayer aussi le deuxième alinéa relatif à l'article 18 touchant la négociation par une association non reconnue.

(17) Un fait illustre cette situation. Au début de décembre 1958, la Commission de relations ouvrières prononça la dissolution d'un local d'une < union internationale ». (Voir Procès-verbal C.R.O., 1959, pp. 720 et suivantes.) Trois mois plus tard, au début de mars, cette union réorganisa son local dans le même atelier et le mois suivant, fin d'avril 1959, lui émit une charte nouvelle. En juin ce local demanda d'être reconnu par la Commission de relations ouvrières. Et la Commission dû le 6 août, lui émettre un nouveau certificat de reconnaissance syndicale. (Voir Procèsverbal C.R.O., 1959, p. 553.) On voit là, la différence de statut devant la Commission entre une association de fait, non incorporée, sans autonomie, sans officiers responsables et subordonnée à une centrale, et le statut du syndicat incorporé en vertu de la Loi des syndicats professionnels pour lequel la dissolution prononcée par la Commission est définitivement fatale. 